\title{
A case of metachronous biliary carcinoma
}

\author{
M I M De Zoysa ${ }^{1}$, A S Dharmawardana ${ }^{2}$, A U Abayadeera ${ }^{1}$, A A Pathirana ${ }^{3}$
}

\section{Introduction}

There have been a few case reports of metachronous gallbladder and extrahepatic bile duct (EHBD) carcinoma, especially associated with an anomalous junction of the pancreaticobiliary duct system. Double common bile duct (DCBD) is a rare congenital malformation. We report a case of a distal bile duct carcinoma presenting two years after radical cholecystectomy for gallbladder carcinoma in a patient with a DCBD.

\section{Case report}

A 45-year old man was incidentally found to have a gallbladder mass on ultrasound examination. He had no history of jaundice, loss of appetite or loss of weight. Contrast enhanced CT of the abdomen showed a $3 \mathrm{~cm}$ polypoidal gallbladder tumour without evidence of invasion (Figure 1). There was no liver metastasis or ascites. He underwent open cholecystectomy because of the possibility of gallbladder cancer. Histology showed a well differentiated adenocarcinoma with involvement of the serosal margin. Ten days later he underwent a wedge resection of the gall bladder bed and resection of porta hepatis lymph nodes. Histology showed no evidence of residual tumour either in the resected liver or the lymph nodes. He was followed up regularly at the outpatient clinic with clinical assessment, abdominal ultrasonography and liver function tests.

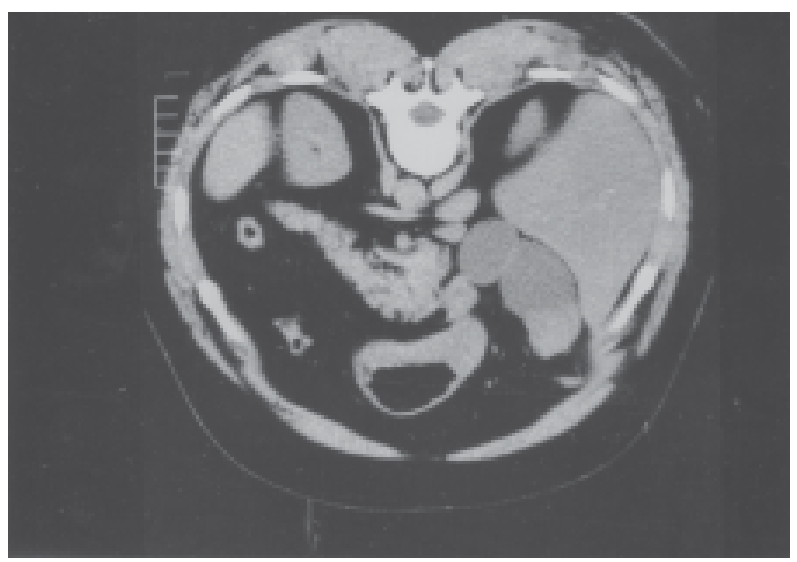

Figure 1. CT showing a tumour in the fundus of the gallbladder.
Two and a half years later he presented with progressive obstructive jaundice and loss of appetite. A CT scan showed intrahepatic and extrahepatic bile duct dilatation along with dilatation of the main pancreatic duct with irregular tapered narrowing distally in the head of the pancreas. The head of the pancreas appeared bulky, but the enhancement was uniform excluding a pancreatic head tumour. An ERCP confirmed the presence of extra and intrahepatic bile duct dilatation and also showed ulceration of the papilla. There was no evidence of an anomalous pancreatico-biliary junction (long common channel). A biopsy taken from within the papilla during the procedure did not reveal any dysplasia or malignancy. The common bile duct was stented with a $10 \mathrm{Fr}, 10 \mathrm{~cm}$ plastic stent.

A repeat ERCP performed 2 months later showed an ulcerative growth at the ampulla encircling the stent and a biopsy confirmed it to be a moderately differentiated adenocarcinoma. A repeat CT abdomen suggested a periampullary carcinoma (Figure 2). The gall bladder bed and the porta hepatis were free of tumour, excluding a locoregional recurrence.

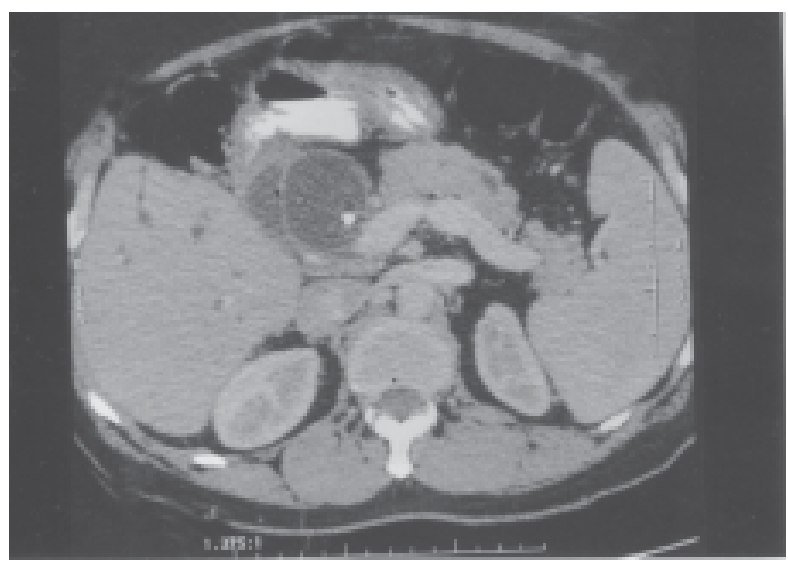

Figure 2. CT showing a double CBD with a stent in the larger duct.

The patient underwent a pancreatico-duodenectomy. During surgery imprint cytology was performed from the cut end of the pancreas to confirm tumour clearance. A double common bile duct (DCBD) was identified. The larger common bile duct which contained the biliary stent was

${ }^{1}$ Department of Surgery, Faculty of Medicine, University of Colombo, ${ }^{2}$ Surgical Unit, National Hospital, and ${ }^{3}$ Department of Surgery, Faculty of Medical Sciences, University of Sri Jayewardenepura, Sri Lanka.

Correspondence: MIMdeZ, e-mail <ishandz@hotmail.com>. Received 16 October 2009 and revised version accepted 22 April 2010. Competing interests: none declared. 
seen to be opening into the major duodenal papilla. The smaller accessory bile duct (ACBD) was opening into the first part of the duodenum. Bile flow was noted from both ducts which were anastomosed separately to the jejunal loop. He had an uneventful recovery.

The histology of the pancreatico-duodenectomy specimen confirmed a moderately differentiated adenocarcinoma arising from the lower CBD without any lymph node deposits. He was referred to the oncologists as the tumour extended to within $1 \mathrm{~mm}$ of the pancreatic resection margin.

\section{Discussion}

Although multiple cancers are not uncommon in the colon, pancreas and oesophagus, multiple bile duct carcinomas are rare [1]. The occurrence of coincidental multiple bile duct carcinoma is unlikely as biliary carcinomas are very rare with a prevalence of $<0.1 \%$ in autopsies. It is tempting to speculate that these patients have a predisposition to multiple biliary carcinomas. Some of these multiple tumours are associated with pancreaticobiliary maljunction [2]. This patient had a DCBD. DCBD is a rare congenital anomaly in which two common bile ducts exist. One usually has normal drainage into the papilla duodeni major and the other usually named the ACBD opens in different parts of the upper gastrointestinal tract (stomach, duodenum, ductus pancreaticus or septum). This anomaly is of importance since it is often associated with biliary lithiasis, choledochal cyst, anomalous pancreaticobiliary junction (APBJ) and upper gastrointestinal tract malignancies [3]. The incidence and type of complicating cancer varies according to the site of the opening of the ACBD. Concomitant gastric cancer was frequently noted when the ACBD opened into the stomach, whereas cancer of the biliary system was common when the ACBD opened into the second portion of the duodenum or the pancreatic duct [4]. Although a few tumours associated with this rare anomaly have been reported, metachronous tumours have not.

In the diagnosis of metachronous tumours it is important to exclude the possibility of the second tumour being a metastasis of the first [5]. In this patient the surgical margins of the gallbladder tumour were negative for cancer. There was no possibility of a distal bile duct carcinoma being missed at the time of initial diagnosis as the second carcinoma was diagnosed nearly two years after the first. The two tumours were histologically distinct with the gall bladder tumour being well differentiated whereas the distal bile duct tumour was moderately differentiated. These facts indicate that the two tumours were truly metachronous.

The origin of DCBD could be related to a random subdivision of the hepatic diverticulum during the first week of embryogenesis or due to differences in recanalisation of the hepatic primodium [6,7]. In this patient DCBD was suspected on CT and confirmed during surgery for the cholangiocarcinoma in the distal common bile duct. It was not detected on CT or during surgery when he initially presented with a carcinoma of the gall bladder probably because the biliary system was not dilated at that time.

\section{References}

1. Ogawa A, Sugo H, Takamori S, Kojima K, et al. Double cancer in the common bile duct: molecular genetic findings with an analysis of LOH. Journal of Hepatobiliary and Pancreatic Surgery 2001; 8: 374-8.

2 Komi N, Takehara H, Kunitomo K. Choledocal cyst: anomalous arrangement of the pacreatico-biliary ductal system and biliary malignancy. Journal of Gastroenterology and Hepatology 1989; 4: 63-74.

3 Djuranovic SP, Ugljesic MB, Mijalkovic NS, et al. Double common bile duct: a case report. World Journal of Gastroenterology 2007; 13: 3770-2.

4 Yamashita K, Oka Y, Urakami A, Iwamoto S, Tsunoda T, Eto T. Double common bile duct: a case report and a review of the Japanese literature. Surgery 2002; 131: 676-81.

5 Merenda R, Portale G, Sturniolo GC, Marciani F, Faccioli AM, Ancona E. A rare surgical case of metachronous double carcinoma of the biliary tract. Scandinavian Journal of Gastroenterology 2007; 42: 1265-8.

6 Knisely AS. Biliary tract malformations. American Journal of Medical Genetics 2003; 122: 343-50.

7 Nakamura K, Mitsubuchi H, Miyayama H, Yatsunami K. Complete absence of the bile and pancreatic ducts in a newborn: a new entity of congenital anomaly in hepatopancreatic development. Journal of Human Genetics 2003; 48: 380-4. 